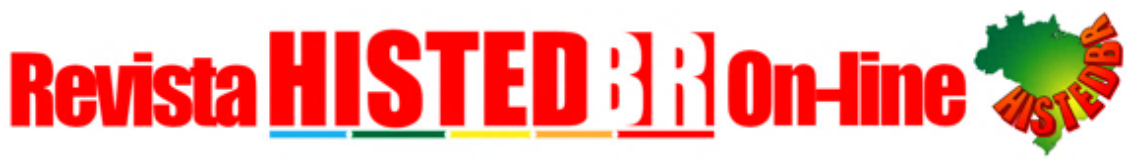

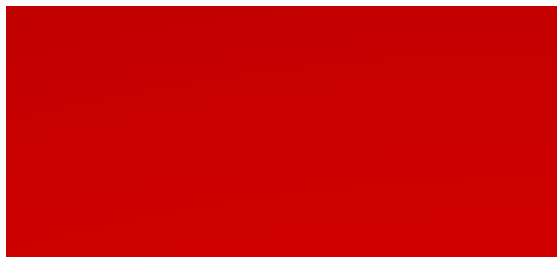

A Revista HISTEDBR On-line publica artigos resultantes de estudos e pesquisas científicas que abordam a educação como fenômeno social em sua vinculação com a reflexão histórica

Correspondência ao Autor Nome: Germán Hours E-mail: gerhours22@gmail.com Instituição: Universidad Nacional de La Plata, Argentina

Submetido: $11 / 10 / 2020$

Aprovado: 12/09/2021

Publicado: 14/12/2021

doi> 10.20396/rho.v21i00.8661580 e-Location: e021055 ISSN: $1676-2584$

Como citar ABNT (NBR 6023): HOURS, G. La evaluación y la matematización del sujeto. El tú puedes en el discurso pedagógico. Revista HISTEDBR On-line, Campinas, SP, v. 21, p. 1-17, 2021. DOI:

10.20396/rho.v21i00.8661580.

Disponível em:

https://periodicos.sbu.unicamp.br/o js/index.php/histedbr/article/view/8 661580. Acesso em: 14 dic. 2021

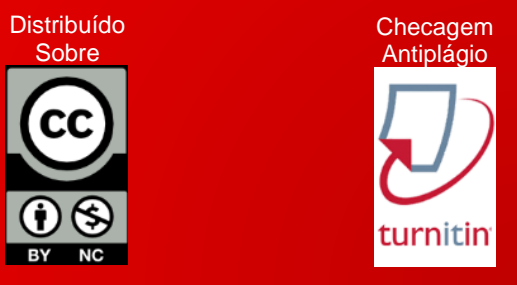

\section{LA EVALUACIÓN Y LA \\ MATEMATIZACIÓN DEL SUJETO. EL TÚ PUEDES EN EL DISCURSO PEDAGÓGICO}

\author{
Q Tattes Germán Hours* \\ Universidad Nacional de La Plata
}

\section{RESUMEN}

La formación ciudadana a la cual responde la educación, como bien señaló Michel Foucault (1985), requiere de generar sujetos dóciles y productivos que internalicen y se apropien de los requerimientos de la sociedad capitalista, naturalizando los mandatos sociales, como un producto lógico, pero también correspondiente a un orden natural, para garantizar el buen funcionamiento del sistema de vida. En este contexto la evaluación ha jugado un papel extraordinario. Sirviendo como herramienta de estandarización y clasificación institucional ha tenido la función de legitimar ciertas ideas que son instituyentes del sistema capitalista. Siguiendo estas ideas, en este artículo, se presentan algunas perspectivas teóricas que permitan profundizar en estas cuestiones, estableciendo un corrimiento del discurso tradicional, para posibilitar una mirada política, crítica y amplia sobre el tema.

PALABRAS CLAVE: Evaluación. Control. Capitalismo. Rendimiento. 


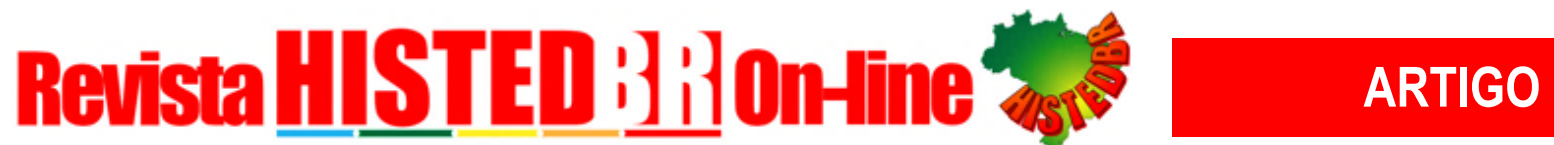 \\ THE EVALUATION AND MATHEMATIZATION OF THE SUBJECT. THE YOU CAN IN THE PEDAGOGICAL DISCOURSE
}

\begin{abstract}
The citizen training to which education responds, as Michel Foucault (1985) pointed out, requires generating docile and productive subjects who internalize and appropriate the requirements of capitalist society, naturalizing social mandates, as a logical product, but also corresponding to a natural order, to guarantee the proper functioning of the life system. In this context, evaluation has played an extraordinary role. Serving as a tool for institutional standardization and classification, it has had the function of legitimizing certain ideas that are instituting the capitalist system. Following these ideas, in this article, some theoretical perspectives are presented that allow us to delve into these questions, establishing a shift in traditional discourse, to enable a politic, critical and broad look at the subject.
\end{abstract}

Keywords: Evaluation. Control. Capitalism. Performance.

\section{A AVALIAÇÃO E MATEMATIZAÇÃO DA DISCIPLINA. O VOCÊ PODE NO DISCURSO PEDAGÓGICO}

\section{Resumo}

A formação cidadã à qual a educação responde, como apontou Michel Foucault (1985), requer a geração de sujeitos dóceis e produtivos que internalizem e se apropriem das exigências da sociedade capitalista, naturalizando mandatos sociais, como produto lógico, mas também correspondendo a uma ordem natural, para garantir o bom funcionamento do sistema de vida. Nesse contexto, a avaliação tem desempenhado um papel extraordinário. Servindo como instrumento de padronização e classificação institucional, teve a função de legitimar certas ideias que estão instituindo o sistema capitalista. Seguindo essas ideias, este artigo apresenta algumas perspectivas teóricas que nos permitem aprofundar essas questões, estabelecendo um deslocamento do discurso tradicional, para possibilitar uma visão política, crítica e ampla sobre o assunto.

Palavras-chave: Avaliação. Controle. Capitalismo. Desempenho. 


\section{Revista HISTED Bifilontine ARrico}

\section{EVALUACIÓN O LA MATEMATIZACIÓN DEL SUJETO}

Las prácticas de la evaluación no son nuevas, como tampoco lo son las costumbres que tienen las instituciones de aplicar con rigurosidad los sistemas formales de evaluación y el valor que a ella se le otorga en todo proceso, no sólo educativo.

En plena Edad Media, la Compañía de Jesús, fundada por San Ignacio de Loyola, ya utilizaba un sistema combinado de informes y notas de las actividades y el potencial de cada uno de los jesuitas que predicaban la religión por todo el mundo, en una época en la cual los navíos de vela eran la única forma de transporte y de comunicación. (CHIAVENATO, 2007, p. 242).

Su desarrollo se remonta a mucho tiempo atrás, pero en la actualidad ha alcanzado niveles extraordinarios, incluyendo análisis y criterios de verdad permanentes sobre los objetos, los sistemas, y, en especial, sobre los sujetos; claro, "[...] en la Modernidad, el conocimiento del mundo es virtualmente su conquista, [pero] cuya racionalidad sólo puede provenir de la acción del hombre sobre él.” (INNERARITY, 1987, p. 109). En el terreno educativo, ya a principios del siglo XX, se propiciaron movimientos en concordancia con cierta teoría de la administración del saber, cuyo propósito era la optimización de la capacidad de enseñanza del docente y de aprendizaje del alumno, con concepciones que veían la educación como una maquinaria que podía ser perfeccionada hasta la máxima posibilidad, piénsese en el fordismo o el taylorismo como ejemplo de esa para entonces nueva forma de operar sobre lo educativo. En este contexto, la educación comenzó a correr mucho más pronunciadamente en paralelo con el mundo y la fuerza de trabajo del ser humano, estableciendo un cálculo de precisión y vínculo, hasta hoy permanente, con la potenciación del rendimiento individual y la producción. Es el comienzo de una educación pensada desde un aprovechamiento del consumo de energía y el tipo de ambiente ideal para el funcionamiento del sistema capitalista, algo considerado una optimización de los tiempos de formación. Es así que la evaluación cobra una especial importancia en el consiguiente planteamiento mecanicista de la administración de la educación para resolver el problema de la eficiencia del sistema educativo mismo.

Desde la Revolución Industrial, paulatina, pero constantemente, el sistema capitalista comprendió que se podía optimizar la tarea humana del mismo modo que fue perfeccionando y corrigiendo la variable máquina. Este es el momento en el que la evaluación se vinculó de manera definitiva e instituyente con el sistema económico-productivo en el campo de la educación. Justamente con el advenimiento de la Modernidad, comenzó una etapa de reconfiguración total de los planteamientos de vida y producción, que engendró un sistema educativo en el que el principal interés se enfocó en la formación del sujeto para su adecuación a ese sistema que ubicó a la máquina y a la industria como forma de vida. En este sentido, Idalberto Chiavenato (2007, p. 243) afirma que "[...] las mismas cuestiones que habían sido planteadas respecto a la máquina fueron transferidas a las personas [...]”, en todos los órdenes del nuevo mundo; explicando este autor, que esta forma de concebir la 


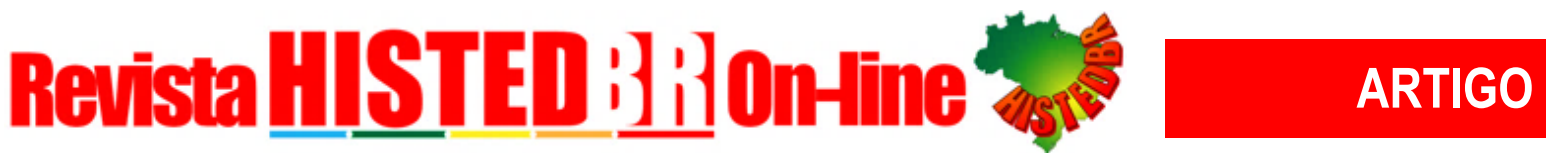

educación surgió a partir de una serie de preguntas que se planteaban: “¿Cómo conocer y medir el potencial de las personas? ¿Cómo conseguir que aprovechen todo ese potencial? ¿Cuál es la fuerza básica que impulsa su energía hacia la acción?” (CHIAVENATO, 2007, p. 243). Para Chiavenato, estos interrogantes dieron lugar a infinidad de respuestas trasladables al sistema educativo, que provocaron "[...] el surgimiento de técnicas administrativas capaces de crear las condiciones para que el desempeño humano en las organizaciones mejorará efectivamente, así como toda una avalancha de teorías respecto a la motivación para el trabajo." (CHIAVENATO, 2007, p. 243).

Desde la misma gestación, cuyo proceso también es evaluado, el sujeto transcurre su vida siendo permanentemente sometido a incontables mecanismos de evaluación. Geo Saura (2016, p. 13) sostiene que esta lógica es así porque "[...] las formas de gobierno contemporáneas requieren sujetos que cuantifiquen la conducción de sus conductas y sean controlados mediante el poder de los números y el juicio de la evaluación." Agregando además, que se trata de una forma de poder que subyuga, somete, pero también, define los sentidos, cuanto más, los trayectos particulares, en definitiva, los destinos de cada quien.

El sujeto está sujetado al número. En ningún periodo histórico anterior los números han tenido la validez que poseen actualmente. Nunca lo numérico ha ostentado la capacidad que hoy en día posee para sustentar argumentos y práctica políticas concretas orientadas al control y a la construcción de sujetos. El número tiene la capacidad de sujetarnos a él, de construirnos. El resultado: sujetos en procesos de sujeción a lo numérico. Nikolas Rose (1999) acertó al afirmar que vivimos en una época en la que somos «gobernados por los números». Las prácticas para clasificarnos, compararnos y categorizarnos son medios por los que somos gobernados por lo numérico (Ozga, 2008). (SAURA, 2016, p. 12).

En definitiva, al ritmo del progreso y la industrialización, las sociedades modernas han desarrollado con suma amplitud y eficacia un conjunto de dispositivos que permitieron lo que este autor denomina las “[...] sociedades de la auditoría." (SAURA, 2016, p. 13).

Lo cierto es que la evaluación es una apreciación sistemática acerca del rendimiento de cada sujeto en su desempeño, y de su potencial desarrollo y alcances a futuro. "Toda evaluación es un proceso para estimular o juzgar el valor, la excelencia y las cualidades de una persona [...]" (SAURA, 2016, p. 243), o de su condición para la tarea, razón por la cual no se circunscribe al ámbito educativo, teniendo alcances a todas las dimensiones del sistema que estructura a una sociedad. En este sentido, Saura (2016, p. 243) describe que

[...] la evaluación de los individuos que desempeñan papeles dentro de una organización se hace aplicando varios procedimientos que se conocen por distintos nombres, como evaluación del desempeño, evaluación de méritos, evaluación de los empleados, informes de avance, evaluación de la eficiencia en las funciones, etc. 


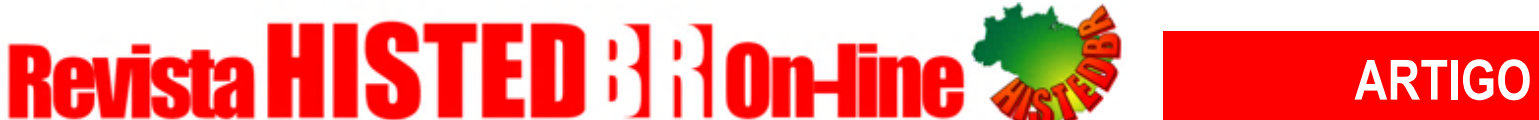

Fiel reflejo de la ciencia experimental moderna, es una operatoria para estandarizar al sujeto, requiriendo para ello de la matematización de sus conductas; su tarea es formular, controlar y decidir acerca de las propiedades del sujeto del mismo modo a como se hace con los objetos, pero como las supuestas propiedades matemáticas de los sujetos no son observables, requiere de toda una construcción teórica que respalde esas acciones, siendo allí donde cobra valor el discurso pedagógico, que tributando a esa ciencia moderna que el capitalismo puso en relieve, terminó configurando un mundo de certezas sobre un sujeto cuantificable, precisamente para intervenir sobre éste de una manera calculada, pero que al mismo tiempo pudiera ser enunciada desde un orden natural. Desde esta nueva perspectiva que instaló la Modernidad, la evaluación

[...] adquiere una importancia metodológica decisiva en orden a la constitución y garantía de la objetividad. Pero este garantizar, tiene que ser un cálculo, porque sólo la calculabilidad garantiza de antemano y constantemente que se tenga la certidumbre de lo que se quiere representar. El representar [...] es un proceder desde sí en el dominio de lo asegurado que previamente es preciso asegurar. (INNERARITY, 1987, p. 108).

Por lo tanto, y esto no es casual, del mismo modo a como se configura la ciencia experimental, la evaluación

[...] en su forma ideal, es un experimento [que obtiene valor] sólo cuando se observen los efectos de variaciones controladas de una propiedad llamada 'operativa' en otra propiedad llamada 'experimental', mientras mantiene constantes todas las otras propiedades que podrían influir sobre la segunda. (MARRADI; ARCHENTI; PIOVANI, 2007, p. 18).

En definitiva, toda evaluación implica una forma experimental de hallar el cálculo matemático posible, más abarcativo y generalizador que posibilite la nominación del sujeto dentro de los estándares institucionalmente establecidos por el sistema que lo comprende, es decir, en términos macro, el sistema capitalista.

\section{LA AUTOEVALUACIÓN: HACIA LA PERFECCIÓN DEL DISCIPLINAMIENTO Y CLASIFICACIÓN}

Ahora bien, esta forma calculada de cuantificar la conducta humana tiene algunos límites. Como señalan Alberto Marradi, Nelida Archenti y Juan Ignacio Piovani (2007) para analizar la metodología de la ciencia experimental en el campo de las ciencias sociales,

[...] los límites prácticos consisten en que no siempre es técnicamente posible controlar a la perfección las variaciones en la propiedad operativa, y todavía menos posible es neutralizar perfectamente la influencia de las propiedades que se deberán mantener efectivamente constantes [...]. (MARRADI; ARCHENTI; PIOVANI, 2007, p. 19). 


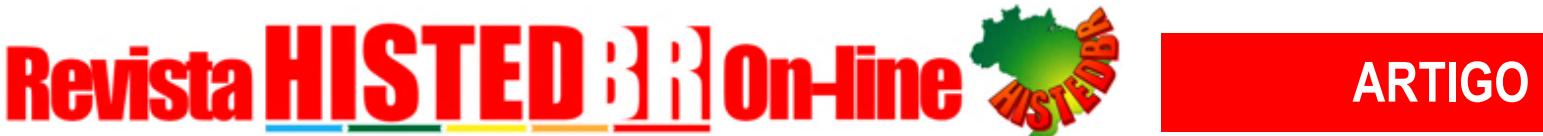

Dado que el sujeto en este campo tiene un valor superlativo y su generalización sería una incongruencia. En consecuencia, el saber pedagógico, entendiendo la complejidad que implica intervenir sobre las relaciones sociales y las subjetividades que éstas comprenden, se ocupó en las últimas décadas por indagar sobre las posibles combinaciones que permitieran ejercer influencias para lograr la función matemática que la evaluación demanda, presentadas como una manera socialmente más significativa.

María Amparo Calatayud Salom (2008), afirma que

[...] una de las estrategias que puede contribuir a afrontar la diversidad de los discentes en el aula es enseñarles a que evalúen su propio aprendizaje. La autoevaluación puede y debe ser un instrumento que facilite atender, respetar y valorar los distintos ritmos de aprendizaje según las diferentes características del alumno.

Considerando que mediante esta forma de evaluación, se tienen en cuenta las " $[\ldots]$ características relativas, por ejemplo, a: capacidades, estilos de aprendizaje, estrategias cognitivas, experiencias y conocimientos previos, motivación, atención, ajuste emocional y social, etc [...]" (CALATAYUD SALOM, 2008), que con los métodos tradicionales, no son alcanzados. Por lo tanto, bajo estas perspectivas supuestamente novedosas, la utilización de la autoevaluación, que supone un modo más significativo de que cada sujeto dimensione su rol y su participación en su propia construcción como ciudadano, supone una mayor eficiencia y alcance, puesto que toma en cuenta indicadores que se consideran dan cuenta de variables de la subjetividad que en las formas tradicionales quedan desentendidos, estableciendo diversos análisis que le proporcionan a la organización educativa también una supuesta mayor eficacia. En los modos educativos, la autoevaluación supone un acuerdo mediante consenso de partes: docente, alumno e institución. Para muchas tendencias progresistas, ésta simbolizó un avance en los procesos de enseñanza, definiéndose como una administración participativa e inclusiva de la educación. En esta operatoria, tanto los contenidos, como los objetivos y las estrategias para abordar la enseñanza, pero sobre todo el aprendizaje, son presentados como una formulación conjunta que se genera luego de una auténtica negociación entre todas las partes. Esta negociación que supone establecer una relación de mayor igualdad entre docente y alumno, que es regida siempre por la institución que los comprende, es pensada como una fuente de incentivo y de representación de una educación más adecuada a los intereses de los alumnos. Sin embargo, por ejemplo, Blanca Bolaños Retavisca et al. (2019, p. 4), que presentaron los resultados de un estudio que buscó identificar el papel que juega la memoria en los procesos de enseñanza y evaluación del aprendizaje, describen que

[...] en el estudio '¿Qué pensamos cuando evaluamos? La evaluación al tablero' de Herrera, Gámez, Torres, Corredor \& Quintero (2008), [...] para los maestros participantes la calificación es una prioridad convirtiéndose 'en una medida del conocimiento adquirido por los estudiantes, además de ser requisito exigido institucionalmente'. 


\section{Revista HISTED : Iil Onrline}

ARTIGO

Señalando que en ese estudio se afirma que

[...] en las prácticas evaluativas predomina el paradigma conductual en los docentes, y aunque ellos manifiestan que su evaluación está centrada en los procesos, los estudiantes declaran que no conocen los parámetros que se tienen en cuenta en la evaluación, se concluye, pues, que no existe coherencia entre el decir y el hacer del docente. (BOLAÑOS RETAVISCA, et al. 2019, p. 4).

Las últimas décadas, en las que se desarrolló una profusa teoría que pone en relieve al alumno como constructor de su propio saber y futuro, el sistema y las instituciones educativas comenzaron este esquema, como uno avanzado y dinámico, que surge en oposición a la vieja administración por objetivos que provenía de la autoridad escolar, cuestionado por lo traumático que éste es y reconociéndose una arbitrariedad burocrática que, en esencia, dista mucho de corresponderse con los discursos democráticos, que propone una educación más participativa, incluyente y motivadora. Aunque debe reconocerse que la autoevaluación o la supuesta perspectiva abierta hacia la participación del alumno, no deja de ser una arbitrariedad burocrática que se presenta de una manera más disimulada. De esta forma se pone en juego una doble operación: por un lado se hace que el alumno internalice decisiones ajenas que le son presentadas como propias; por otro lado, se hace que el alumno asuma y acepte un compromiso sobre algo que le fue presentado como conveniente para sí mismo, pero que en realidad es conveniente para la institución que lo rige. Lo que ocurre a partir de esta negociación que implicó un supuesto acuerdo entre docente y alumno, es que, existe una relación desigual entre partes que es presentada como con cierta equidad, generando que el alumno asuma un compromiso bajo un supuesto consenso que no es tal, sino que es una forma de coaccionar mediante cierta seducción hacia el compromiso personal del alumno, que termina haciéndose cargo de su propia clasificación y estratificación. Forma que colabora en orientar el desempeño con fines previamente negociados, hacia resultados establecidos previamente. No cabe duda también, que esta forma representa una de bajo costo para que el sistema pueda alcanzar los objetivos deseados. En términos foucaultianos, la evaluación, que es establecida a partir de ciertos límites médicos,

$[\ldots]$ se trata $[\ldots]$ en cuestión del despliegue de un proceso histórico que establecerá una novedosa función por parte del Estado moderno, caracterizada por convertir a la población en un objeto de cálculo y regulaciones, como asimismo, de depuración biológica frente a aquellos elementos anómalos capaces de interferir en su óptimo funcionamiento. (DÍAZ, 2013, p. 5).

Siendo parte del capitalismo, la autoevaluación es parte de un contexto histórico más elaborado signado por un conjunto de transformaciones económicas y políticas producidas por la sociedad neoliberal contractualista, aunque es presentada como " [...] la estrategia por excelencia para educar en la responsabilidad y para aprender a valorar, criticar y a reflexionar sobre el proceso de enseñanza y aprendizaje individual realizado por el discente." (CALATAYUD SALOM, 2008). Tomando un análisis de Martín Díaz, se puede señalar que 


\section{Revista HISTED ilitOn-line}

ARTIGO

de la mano de la autoevaluación se establece " [... . una novedosa función por parte del Estado moderno, caracterizada por convertir a la población en un objeto de cálculo y regulaciones, como asimismo de depuración biológica frente a aquellos elementos anómalos capaces de interferir en su óptimo funcionamiento." (DÍAZ, 2013, p. 5). De esta manera, la evaluación se establece como parte del "[...] despliegue de este poder sobre la vida [que supuso] en la historia de Occidente una profunda transformación de la vida humana al convertirla en un objeto posible de ser manipulado, regulado y modificado de acuerdo con ciertas metas previamente prefijadas.” (DÍAZ, 2013, p. 5).

La gestión educativa moderna, se sostiene sobre estrategias que promueven la formación ciudadana en relación con el buen funcionamiento del sistema productivo, pero asume sus propias falencias, dado que no alcanza a todos por igual, reconociendo la existencia de una población de riesgo, catalogados como grupos vulnerables, que poseen pocas o escasas oportunidades de ser cubiertos por la educación del mismo modo que el resto, mucho menos, de autogestionarse a sí mismos y de contribuir mediante ésta al mejoramiento de su competencia individual para los fines del orden establecido. Con la autoevaluación, se afirma que esto quedaría cubierto, ya que no sólo posibilita al sujeto vulnerable sea comprendido por los beneficios que ésta supone, sino que termina adhiriendo a ciertas conductas virtuosas que lo comprometería directamente con las formas correctas de insertarse en la sociedad civilizada.

Algo que es muy interesante para analizar, es que la autoevaluación en el sistema educativo, es similar a la autoevaluación que se realiza a nivel empresarial. Para Chiavenato (2007, p. 244),

[...] en las organizaciones más democráticas, el propio individuo es el responsable de su desempeño y de su propia evaluación. Esas organizaciones emplean la autoevaluación del desempeño, de modo que cada persona evalúa el propio cumplimiento de su puesto, eficiencia y eficacia, teniendo en cuenta determinados indicadores que le proporcionan el gerente o la organización.

Utilizando el discurso de la democratización y la pluralidad que debe tener una enseñanza de calidad, se le reconoce a esta forma de operar como parte de un conjunto de estrategias que atienden a lo diferente, a la diversidad, puesto que supone tener en cuenta la variabilidad en los ritmos de estudio y de aprendizaje que presentan los alumnos. Desde un trasfondo filosófico y ontológico, la autoevaluación, básicamente consiste en hacer que la condición natural del individuo siga su curso natural, considerándose como una de las formas de laissez faire -dejar hacer- que caracteriza a la autoevaluación, como parte de un sistema que asume al individuo desde una matriz eminentemente natural en el que la necesidad de aprender es propia de su condición humana como especie. En este punto, es necesario recurrir a Pierre Bourdieu (2008, p. 146), quien señaló que

[...] se puede pensar en democratizar la relación pedagógica. Pero yo tengo una serie de indicios que me hacen pensar que mientras más autoritaria es la relación pedagógica, más favorable es para los hijos de las clases 


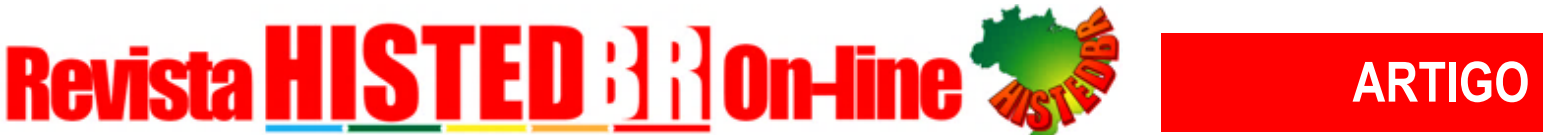

dominadas, mientras que una relación democrática, igualitaria, es favorable a los privilegiados, porque ellos saben cómo dominar una situación de laissez-faire.

Siguiendo esta idea, es insoslayable señalar que este argumento de la desigualdad que en las tendencias más progresistas suele llamarse diversidad-va a ser definido como una consecuencia natural del individuo y por lo tanto algo que caracteriza a la sociedad también de manera natural, por lo tanto, desde esta perspectiva, se desprende la desigualdad como algo natural y no una resultante de una decisión política.

\section{EL DISCURSO DEL TÚ PUEDES. LA LIBERTAD INDIVIDUAL PROYECTADA HACIA LA FELICIDAD}

Entre otras cuestiones, que también intervienen en la autoevaluación, la evaluación se afianza en la razón del sujeto desde el mecanismo que le indica en el tú puedes, que subyace y se antepone a cualquier dispositivo moderno. El tú puedes configura una amplia gama de estrategias vinculadas con la educación de los sujetos, una práctica que conlleva una serie factores que suponen, cada uno de ellos, generarán un cambio de actitud, una mejora en la autoestima y, como consecuencia, un mayor rendimiento en el contexto en el que se encuentre inmerso. Este tú puedes incluso ejerce mayor coacción que el tú debes, y esto es algo que debe reconocérsele al neoliberalismo que, ostentando una superioridad discursiva, el discurso amo, logró maximizar su eficiencia, precisamente, en la falacia de la libertad individual. El tú puedes es un condicionante que es presentado como un estímulo sobre la motivación, que convence al sujeto que la superación es algo que depende exclusivamente de él ${ }^{1}$. Básicamente, aplícase para tratar de generar conductas positivas a partir de reconducir y canalizar sentimientos negativos hacia una acción productiva. Funciona como una fuente de inspiración que genera cierta autoconciencia en el sujeto que lo incita a creer que participar y aprender es poder activar individualmente sobre la materialización de las proyecciones de su vida. No tiene por función sólo generar el acto, sino también el hábito. El tú puedes es la orden que lleva al sujeto a ir tras la seductora promesa de la felicidad, pero, es evidente que para que éste se vea seducido por esa promesa y accione en ese sentido, tuvo que haber antes una acción educativa que desarrolle en éste la capacidad de considerarla como tal: entra a jugar aquí la Pedagogía, y la acción pedagógica, como herramienta fundamental del capitalismo. Byun-Chul Han (2014, p. 12) señala al respecto que, "[...] el tú puedes produce coacciones masivas en las que el sujeto del rendimiento se rompe en toda regla." Han señala además que la coacción propia del tu puedes es más fatal que la coacción ajena que implica el tú debes, porque no es posible ninguna resistencia contra una orden que provenga de uno mismo. Cuanto más efectiva es la disciplina cuando es el propio sujeto quien la regula en sí mismo. Prepararse para ser evaluado, aceptar ser evaluado y responder positivamente y condescendientemente a la revelación de sí mismo que ese ser evaluado a determinado, es en acto, el mismo sujeto 


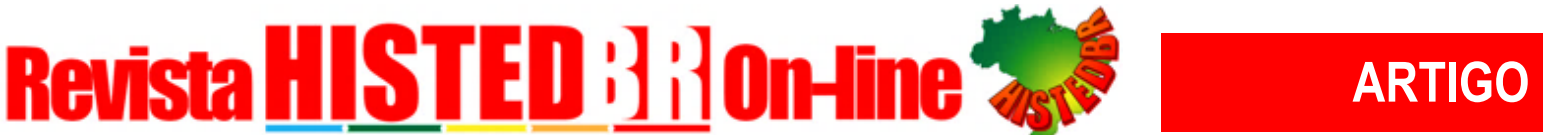

administrandose a sí mismo ese cierto poder disciplinario oculto que la evaluación y los/as evaluadores ejercen sobre él. Es el resultado que demuestra la pedagogización del sujeto. De esta manera, la evaluación implica un doble mecanismo: por un lado, fiscaliza la promesa de libertad individual y el cumplimiento de esa promesa; por otro lado, hace responsable al sujeto de los resultados que la evaluación determine; puesto que es un mecanismo revestido de objetividad, no es el proyecto ni el evaluador, mucho menos el sistema, es siempre el sujeto el responsable de su propio fracaso.

En las evaluaciones, la mayoría de las veces estandarizadas, es indudable que coexisten mecanismos de gestión de la población y de normalización de las conductas, que sirven para extender lo que Michel Foucault (2012) llamó la gubernamentalidad neoliberal. La evaluación, como sistema -con sus dos actores: el evaluador y el sujeto deseoso de ser evaluado- es el principal agente reproductor del status quo que impone el pensamiento capitalista, en tanto mediante ella se establece un orden jerárquico al que los sujetos han de someterse. Desde esta perspectiva de análisis, la evaluación entonces puede ser resuelta desde tres dimensiones de análisis que definen sus funciones y sus poderes, que son parte de sus grandes activos, aunque no agota en ellas sus posibilidades: por un lado su función o poder selectivo, dada su capacidad de discernir y discriminar para erradicar lo diferente, operando siempre hacia lo instituído respaldando al sistema o institución para la que opera y a la cual responde, legitimando ciertas ideas con sentencia de verdad; por otro lado, su función o poder vinculado con lo aspiracional, es decir, la promesa que le brinda al sujeto de alcanzar un estado superior a partir de su aplicación; y por último, su función o poder punitivo, o su posibilidad de hacer cumplir la norma, en tanto determina y concluye el lugar de cada uno en el escenario social, penalizando mediante la calificación o descalificacion las conductas o potencialidades favorables, como las desviaciones.

No se puede obviar que la evaluación opera para instituir y consolidar el vínculo social o, dicho más precisamente, el modo cómo se establece el contrato social en la Era Moderna. Es presentada como la posibilidad de arbitrar en los problemas con los que la sociedad se encuentra y que, mediante ella, quedan a la espera de una solución. Según consideran Jacques-Alain Miller y Jean-Claude Milner (2004), se ha convertido en el modo de dotar de garantía al objeto que está en juego en las relaciones entre los seres humanos. Nada escapa a lo que ha venido a llamarse el discurso de la evaluación ${ }^{2}$. Así, la evaluación emerge como un fenómeno de civilización que eleva ese contrato al elemento garante del vínculo entre los seres humanos: "[...] es un procedimiento pesado. Esta pesadez surge de la lógica del contrato, no de la lógica de la ley." (MILLER; MILNER, 2004, p. 27). El discurso de la evaluación establece con su metalenguaje órdenes socio-políticas, sustentándose en la base de la constitución de procesos identitarios nuevos, que prometen una resolución efectiva y afectiva de la falta en el sujeto, por medio de la canalización del deseo hacia un significante específico: el consumo.

Al mismo tiempo, la evaluación es un procedimiento que necesita aplicar a todo aquello susceptible de ser evaluado; no puede omitirse entonces el hecho de que es el individuo 


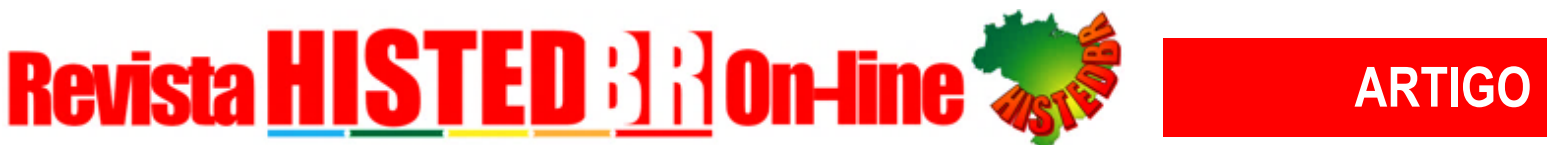

mismo quien consciente o no acepta ser evaluado, pero acepta también que su capacidad evaluadora sea a su vez evaluada en el marco de un proceso formativo. "Ningún sujeto es hoy ajeno a los procesos de evaluación.” (RUIZ ACERO, 2013, p. 235).

Los evaluadores se presentan en nombre de la ciencia [...] es una iniciación y se transmite como una iniciación. Se puede ver como aquello que tienta a la gente, en el sentido de la tentación, de prestarse a la evaluación, diciendo: Una vez que usted será acreditado-evaluado, podrá evaluar a otros. El contenido mismo de la evaluación, de la operación evaluadora, se escapa. Es un cuestionario, entrevistas, este tipo de cosas. Lo más importante es que el otro haya consentido a la evaluación. Consentir a ser evaluado es mucho más importante que la operación de evaluación en sí misma. Digamos incluso: la operación es la de obtener su consentimiento a la operación. (MILLER; MILNER, 2004, p. 31).

En rigor, la evaluación es siempre un proyecto y/o proceso colectivo, marcado por el principio de individualidad del sujeto al que afecta. Es por ello que la filosofía capitalista establecida en los dispositivos de evaluación,

[...] esconde en su estructura coactiva tras la aparente libertad del individuo, que ya no se entiende como sujeto sometido (subject to), sino como desarrollo de un proyecto. Ahí está su ardid. Quien fracasa es, además, culpable y lleva consigo esta culpa dondequiera que vaya. Tampoco hay posibilidad alguna de excusa y expiación. Con ello surge no sólo la crisis de culpa, sino también la de gratificación. (HAN, 2014, p. 12).

Crisis, ambas, que ponen de manifiesto que el sistema capitalista es siempre el endeudador del sujeto;nunca el que saldará la deuda contraída por éste en la resultante, positiva o negativa, de la evaluación. Los logros y los fracasos que la evaluación indique, aunque en el sujeto se haya instalado como un proceso propio, inherente exclusivamente a él, son siempre atribuibles al sistema que la concibió, es por ello que el resultado siempre es favorable al sistema y nunca al sujeto, puesto que mediante ella se miden las posibilidades beneficiosas y los efectos o defectos indeseados para la productividad y el rendimiento. Así operan estas prácticas discursivas que establecen el significante de la evaluación como medio para saciar el deseo del sujeto. De esta manera, retomando a Foucault (1985, p. 141), el sujeto -evaluador o evaluado- queda sometido a "[...] los métodos que permiten el control minucioso de las operaciones [...] que garantizan la sujeción constante de sus fuerzas y les imponen una relación de docilidad-utilidad."

Peter Sloterdijk (1999) presenta la educación y la cultura como técnicas de domesticación del hombre;

[...] reclama una revisión genéticotécnica de la humanidad. En su planteamiento constata que 'las fantasías de selección biopolítica han tomado el relevo de las utopías de justicia' [...] Esta nueva ingeniería 


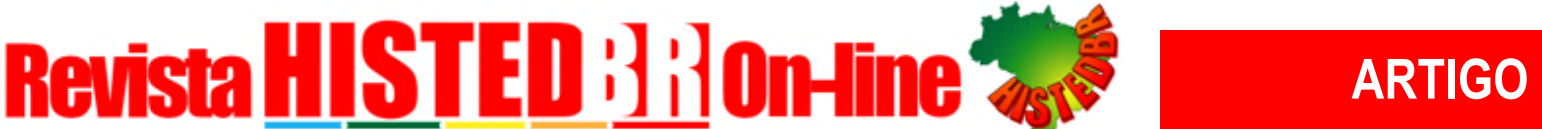

social aparentemente busca cimentarse en una antropología de cuño neodarwinista. (VÁSQUEZ ROCCA, 2009, p. 1-2).

El principio del rendimiento se mide también por la proximidad que las teorías sociales han pregonado, en tanto esa proximidad, que permite la construcción del tejido social, que en la evaluación apunta siempre al vínculo entre los individuos, es un principio que rompe con toda subjetividad posible y, por lo tanto, con el sujeto. Es un principio generalizador y totalizador. Esto, en términos políticos, también es globalización, pero expresado en su mínima unidad. Cuanto más próximos los individuos, más generales son sus estatutos y más proyectables a un sistema y a un mismo deseo se vuelven. El discurso de la igualdad se despoja del sujeto, reponiendo al individuo, totalizándolo a partir de su proximidad con el igual a él, naturalizando una visible entrega absoluta al consumo. El capitalismo elimina toda alteridad posible para someterlo todo al consumo, reduciendo al sujeto a su mera vida biológica. La esencia del capitalismo es la negación de la diferencia. En la diferencia se marca la cultura de la política. La evaluación irrumpe para romper con esa cultura de la diferencia, a favor de la cultura de lo igual. El capitalismo, de esta forma, opera sobre el individuo, para incidir en la población, "[...] entendida como cuerpo múltiple." (VÁSQUEZ ROCCA, 2009, p. 4). Este inconmensurable impacto del pensamiento capitalista sobre el sujeto, "[...] aparece cuando el hombre tiene, técnica y políticamente, la posibilidad no sólo de disponer la vida, sino de hacerla proliferar." (VÁSQUEZ ROCCA, 2009, p. 4). La exaltación hacia el espíritu vital condensa, substancia y enajena al sujeto a su propia naturaleza, puesto que en ella radica su construcción social. Por lo tanto, la evaluación, por definición, está siempre sometida al dictado del rendimiento.

Estos procesos de rendimiento educativo permiten a las agendas políticas educativas ejercer, siguiendo de nuevo a Foucault, esa capacidad de «designar lo que hace entrar a la vida y sus mecanismos en el dominio de los cálculos explícitos y convierte al poder-saber en un agente de trasformación de la vida humana» (Foucault, 173). (VÁSQUEZ ROCCA, 2009. p. 17).

En consecuencia, la evaluación indica que sólo se puede consumir, puesto que cosificando al sujeto también como objeto de ese consumo, como parte del mismo mecanismo, y que, al correr siempre detrás del deseo de consumo, deja se ser, para, fragmentado en diversos objetos que operan en diversos momentos del sistema, se pueda cuantificar ese deseo aun más y garantizar así la perdurabilidad del sistema. Lo que indaga la evaluación no es sobre la moralidad del deseo, sino la relación que se establece entre el sujeto y éste, para verificar los grados de aceptación y compromiso del evaluado con el sistema que lo está evaluando; un Gran Otro, una entidad sin ente que no es Dios -el capitalismo, por ejemplo-, pero que guarda no pocas semejanzas con éste. (LAPLANCHE; PONTALIS, 2004). Esa supuesta libertad del sujeto es la posibilidad de llegar a un acuerdo entre su deseo y esa demanda incesante y dolorosa que nos impone el Gran Otro. 


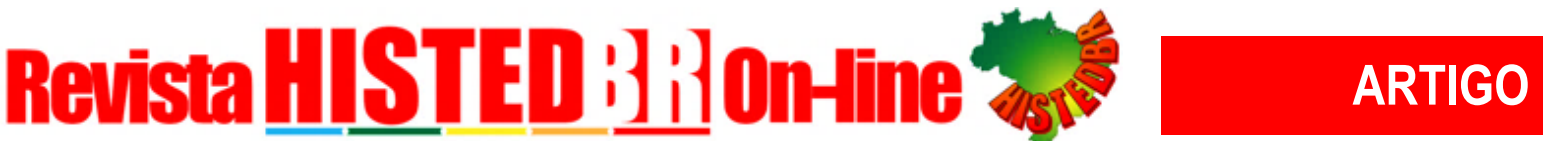

En términos generales, evaluar es obtener información relativa al sujeto, tomándolo como objeto, para ponderar determinadas características, evitar efectos indeseados y actuar de manera adecuada para garantizar la obtención de los resultados deseados en éste. "Si los mecanismos de evaluación permiten medir y controlar lo que a un objeto lo convierte en cosa, ¿por qué no hacerlo con el ser humano, de quien su variabilidad es la gran amenaza de cualquier orden establecido?" (RUIZ ACERO, 2013, p. 234). Por esta razón es que no puede escaparse a la mirada que pone de manifiesto que la evaluación siempre mide el rendimiento, aun cuando se proclame lo contrario. A través de ese deseo de consumo, el sujeto, al ser evaluable en sus conductas y proyectable en sus deseos, pasará de la ilusión de la satisfacción a la queja, manifestada en su plano afectivo, mediante una insatisfacción permanente, que siempre conlleva un relanzamiento del deseo de consumo hacia la meta de la felicidad; he aquí la idea de la autosuperación que tanto proclama el neoliberalismo y que el pragmatismo político/educativo ha reproducido con tanta grandilocuencia. En definitiva, lo que hace tan funcional al capitalismo instalar el deseo de consumo, es que, en términos lacanianos, a diferencia de lo que ocurre con la necesidad, el deseo no puede nunca ser satisfecho ${ }^{3}$.

\section{CONSIDERACIONES FINALES}

La excusa es siempre la complejidad del sistema en el que se vive. Con los mismos argumentos, recurrentemente se justifican todo tipo de acciones proactivas sobre el sujeto. Bajo esa impronta a lo largo de la Modernidad se han ido legitimado incontables mecanismos de gobierno, que limitaron categóricamente los márgenes de manifestación de la sociedad en su totalidad -el sujeto devenido en individuo, considerado de manera general y universal: el sujeto colectivo-. En particular, mediante diversos procesos de cuantificación, es decir, de evaluación, y en nombre del tan proclamado progreso, se han establecido cada vez con mayor énfasis y poder coercitivo tecnologías gubernamentales que han caracterizado a las democracias occidentales, definidas unidireccionalmente en sus discursos hacia el desarrollo de las libertades más avanzadas, instituyendo el condicionamiento de ser siempre, y casi exclusivamente, posibles de ser resueltas por el propio accionar del ser humano. Por supuesto, se hace referencia a la ilusión moderna capitalista de que el logro está al alcance por la mera inicativa individual. Visión moderna de la educación, que respondiendo a cierto progresismo contemporáneo, a decir de Raumar Rodríguez Giménez (2014, p. 136), sólo

[...] se conforma con administrar las libertades, ese pequeño reducto ideológico donde rezuma la miseria de los individuos. El dominio de lo político, modernamente constituido en torno al significante Libertad, que establecía lazo allí donde la contingencia infinita de los cuerpos se hace presente, cede el paso a la necesidad.

Con las instituciones modernas comienza la etapa de constitución de ciertas verdades sobre la vida humana, como así también, las reglas y las formas correctas de acceso a ellas, justificadas siempre en nombre de la ciencia moderna; esa ciencia supuestamente natural, 


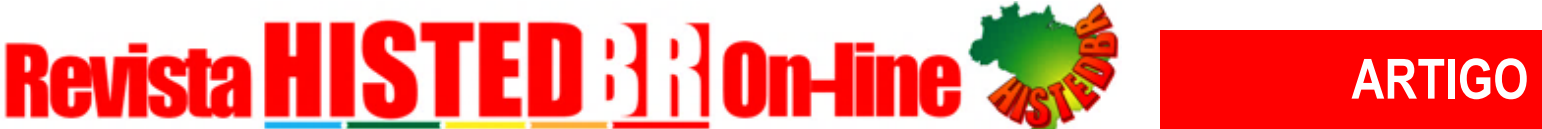

pero que no es otra cosa que una forma de ordenamiento político. Esto posible porque como afirma Mercedes Kerz (2008, p. 17),

[...] no existe organización social que no haya reconocido formas singulares de ordenarse políticamente; fue la democracia la que aportó como novedad la idea de la institución de acuerdos formales y normativos con el objeto de contener la inherente confrontación de las relaciones humanas.

En esta organización, la individualidad y la posibilidad natural que posee el sujeto para ser el artífice de su propio futuro, fue la matriz principal de construcción política y, por ende, de la conformación de las instituciones. Es también la instauración de la idea de que el individuo es un ser social. Una perspectiva política que se revistió de una muy útil razón natural. La constitución individual y las formas de relaciones que, por supuesto, incluye las formas de organización que dieron lugar a las instituciones modernas, siempre fueron aplicadas como una cuestión natural en el ser humano. Al respecto, Kerz sugiere también, que las instituciones se corresponden con algunas de las formas de los acuerdos políticos de la democracia para organizar la convivencia social, y en este punto, la ciencia moderna cobra un papel trascendental; y, aunque marcan con crudeza la posibilidad del sujeto, no se alejan mucho de una configuración ficcional, una especie de puesta en escena, que delimita taxativamente hasta donde puede éste actuar. Lo cierto es que es allí, en las instituciones modernas, donde el sujeto adviene a reconocerse en su estado social y a tomar conciencia de su lugar en el mundo. Es donde se asume un individuo integrante de un colectivo que le otorga sus características tanto generales como particulares; la darwinista idea del ser único e irrepetible, pero con constantes generales de la especie humana que lo comprenden y lo definen. En este contexto que definió sus inconmensurables alcances, la evaluación no deja de ser un objeto de distinción política y discusión teórica, incluso posible de ser sometida a la interpelación más aguda, a pesar de que su carácter habitualmente es revestido de una naturalidad incuestionable, en el afán por dotarla de la inmunidad conceptual que le permita actuar y desplegar su función con libertad, para poder generar los resultados previstos, es decir, para los cuales fue diseñada originalmente en cada caso. Es importante comprender que la evaluación no sólo tiene la función de fiscalizar, sino, una más importante que es generar productos deseados. Existe entonces, una totalidad prescriptiva o concepción uniforme en cada operatoria evaluadora, que siempre subyace y responde al mandato del sistema capitalista.

De esta manera, la autoevaluación, entendida ésta como una variante del dispositivo que es la evaluación en general, se corresponde a un proceso que el docente realiza en relación al quehacer académico institucional, y cuyo fin es promover su mejoramiento; como argumento, se la presenta como una práctica para autoconocimiento que conlleva cambios o transformaciones, pero que fundamentalmente es coherente con los principios, propósitos y funciones de la institución en la que se lleva adelante. Mediante la autoevaluación se personaliza a cada aprendiz, tratando de indagar en los ritmos de su aprendizaje; 


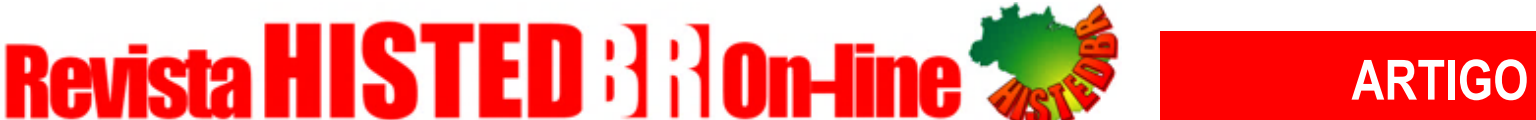

individualizando su fiscalización, que se enmascara bajo cierta forma de consideración y cuidado del sujeto, para observar las debilidades y fortalezas del proyecto institucional, de modo que se puedan proyectar las competencias que debe adquirir para dar respuesta a los requerimientos del plan curricular. La evaluación supuesta desde la objetividad que la conforma, entonces, no es otra cosa que lo que se corresponde con la misma formación que se encuentra para su actividad. Se consolida en la función normativa de la conciencia para plasmar una realidad que es presentada de manera objetiva, por lo tanto, los valores desde donde se sostiene se corresponden con meras estimaciones axiológicas, cuanto más, axiomáticas. La evaluación responde a ese mundo que la Modernidad ha creado en el que ha convertido un producto a la subjetividad, configurando todas sus prácticas y dispositivos tributando a ello, sometiendo así a sus instituciones, a sus costumbres, a su moral y, sobre todo, a su ciencia, en nombre de la naturalidad del ser humano. (FINK, 1976). En nombre de la libertad, la Modernidad instaló paulatinamente a la evaluación como un proceso que supuestamente permite desocultar la verdad, asegurando el dominio del sujeto y, con ello, definiendo la dirección de las acciones de éste y la manera como debe ser vivida la vida. De esta manera, es una herramienta que sirve para una educación que responde a ese

[...] orden político, cuyo principio constitutivo continúa siendo el primado de la subjetividad que discurre en paralelo con una concepción mecanicista de la naturaleza [...] queda planteado en el esquema típico para la justificación del poder: el binomio estado de naturaleza-estado de sociedad [...]. (INNERARITY, 1987, p. 116).

Subsumiendo al sujeto a una sola forma de vida posible: la que impone el sistema capitalista.

\section{REFERENCIAS}

BOLAÑOS RETAVISCA, B. et al. La memoria en la evaluación del aprendizaje en ciencias sociales. Archivos de Ciencias de la Educación, v. 13, n. 15, 2019. DOI: https://doi.org/10.24215/23468866e059. Disponible en: https://bit.ly/31Q9u0U. Acceso en: 29 abr. 2020.

BOURDIEU, P. ¿Qué es hacer hablar a un autor? A propósito de Michel Foucault. In: BOURDIEU, P. Capital cultural, escuela y espacio social. Buenos Aires: Siglo XXI, 2008.

CALATAYUD SALOM, M. A. La autoevaluación como estrategia de aprendizaje para atender a la diversidad. Educaweb. Departamento de Didáctica y Organización Escolar. Facultad de Filosofía y Ciencias de la Educación. Universitat de Valencia. 2008. Disponible en: https://bit.ly/2Z17YvT. Acceso en: 20 mayo 2020.

CHIAVENATO, I. Evaluación del desempeño. En Administración de Recursos Humanos. México: McGraw-Hill, 2007. p. 241-269. 


\section{RevistafilSTED BilOnHine ARTICo}

DÍAZ, M. Del disciplinamiento de los cuerpos al gerenciamiento de la vida. Mutaciones biopolíticas en el presente en torno a la construcción de la anormalidad. De Prácticas y Discursos. Cuadernos de Ciencias Sociales, año 2, n. 2, 2013. Resistencia: Universidad Nacional del Nordeste - Centro de Estudios Sociales. ISSN 2250-6942.

FINK, E. La filosofía de Nietzsche. Madrid: Alianza, 1976.

FOUCAULT, M. El poder, una bestia magnífica. Buenos Aires: Siglo XXI, 2012.

FOUCAULT, M. Vigilar y castigar. El nacimiento de la prisión. México: Siglo XXI, 1985.

HAN, B-C. La agonía del eros. Traducción Raúl Gabás. Barcelona: Herder Editorial, 2014. ISBN 978-84-254-3255-2.

INNERARITY, D. Modernidad y postmodernidad. Anuario Filosófico, v. 20, p. 105-129, 1987. Depósitop Académoco Digital, Universidad de Navarra. Disponible en: https://hdl.handle.net/10171/2278. Acceso en: 21 mayo 2020.

KERZ, M. Dominio político: permanencias y cambios. Aportes para una reflexión teórica. Buenos Aires: Teseo, 2008. ISBN: 978-987-1354-26-9.

LAPLANCHE, J.; PONTALIS, J.-B. Diccionario de psicoanálisis. Buenos Aires: Paidós, 2004.

MARRADI, A.; ARCHENTI, N.; PIOVANI, J. Metodología de las Ciencias Sociales. Buenos Aires: Emecé, 2007.

MILLER, J.; MILNER, J. ¿Desea usted ser evaluado? Málaga: Miguel Gómez, 2004.

RODRÍGUEZ GIMÉNEZ, R. Por una lectura política de la relación cuerpo-educaciónenseñanza. Polifonías Revista de Educación, año III, n. 5, p 128-143, 2014. Disponible en: https://bit.ly/3zFFxW2 Acceso en: 17 feb. 2020.

RUIZ ACERO, I. Reseña de Miller y Milner (2004) ¿Desea usted ser evaluado? Athenea Digital, v. 13, n. 1, p. 233-236, 2013. Disponible en https://bit.ly/3uaHbxJ. Acceso en: 15 mayo 2019.

SAURA, G. Neoliberalismo como discurso la evaluación en educación entre tecnologías políticas neoliberales y la nueva filantropía. Revista Educação, Sociedade \& Culturas, v. 47, p. 11-30, 2016.

SLOTERDIJK, P. Reglas para el parque humano. Una respuesta a la "Carta sobre el Humanismo". Conferencia pronunciada en el Castillo de Elmau, Baviera, en julio de 1999, con motivo del Simposio Internacional "Jenseits des Seins / Exodus from

Being / Philosophie nach Heidegger", en el marco de los Simposios del Castillo de Elmau. Traducción: Fernando La Valle. 1999. 


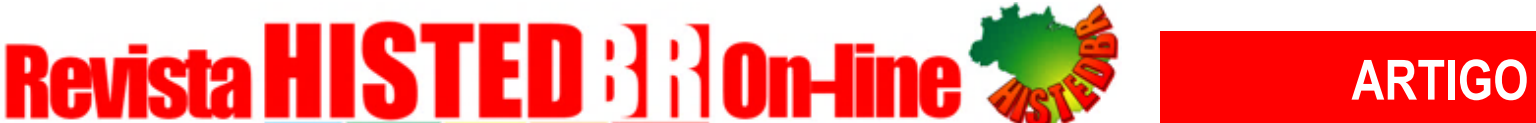

VÁSQUEZ ROCCA, A. Sloterdijk, Agamben y Nietzsche: biopolítica, posthumanismo y biopoder. Nómadas: Critical Journal of Social and Juridical Sciences, v. 23, n. 3, 2009. Euro-Mediterranean University Institute Roma, Italia. Publicación Electrónica de la Universidad Complutense. Disponible en: https://bit.ly/3zzLQuo. Acceso en: 14 jul. 2020.

\section{AUTORIA:}

* Magíster en Deportes de la Universidad Nacional de La Plata (UNLP, Argentina). Profesor de Educación Física y Deportes, Universidad Nacional de La Plata (UNLP, Argentina). Contacto: gerhours22@gmail.com

COMO CITAR ABNT:

HOURS, G. La evaluación y la matematización del sujeto. El tú puedes en el discurso pedagógico.

Revista HISTEDBR On-line, Campinas, SP, v. 21, p. 1-17, 2021. DOI:

10.20396/rho.v21i00.8661580. Disponível em:

https://periodicos.sbu.unicamp.br/ojs/index.php/histedbr/article/view/8661580. Acesso em: 14 dic. 2021.

\section{Notas}

\footnotetext{
${ }^{1}$ No es casual que este condicionante determinado por el tú puedes funcione de manera similar a un eslogan; los vínculos entre la educación y el sistema de consumo del mundo capitalista son más que visibles. Frases como you can do it, u otras más conocidas como just do it -eslogan de la marca Nike-, que siempre apuntan a la conducta del sujeto, al ser tan contundentes y al mismo tiempo tan sintéticas, hace que sean recordadas rápidamente, activando de inmediato los mecanismos internos para generar una respuesta determinada de manera casi automática. Al igual que en un eslogan, en el que una frase corta y pegadiza sistematizada logra generar un gran impacto en el sujeto, este tipo de frases también son interiorizadas por el sujeto de manera que logra generar una modificación en sus conductas, aunque a diferencia de un eslogan, es presentada como una autosuperación personal e individual, nunca relacionada a una situación de consumo, sino educativa.

2 Para Miller y Milner, “[...] el «discurso de la evaluación» es [...] el siervo del discurso del capitalismo, pues los procesos del primero consisten en producir objetos de consumo válidos para el segundo. Se trata de que todo objeto que sirva para el consumo entre en el mercado después de haberse evaluado las condiciones que permitan gozar al sujeto de ese objeto." (RUIZ ACERO, 2013, p. 233).

${ }^{3}$ Para Lacan, “[...] el deseo nace de la separación entre necesidad y demanda; es irreductible a la necesidad, puesto que en su origen no es relación con un objeto real, independiente del sujeto, sino con la fantasía; es irreductible a la demanda, por cuanto intenta imponerse sin tener en cuenta el lenguaje y el inconsciente del otro, y exige ser reconocido absolutamente por él." (LAPLANCHE; PONTALIS, 2004, p. 97).
} 